\title{
Construction of an Efficient In Vitro System for Analysis of Transcription from Sigma 54-Dependent psp $A$ Promoter
}

\author{
Kook Han, Geunu Bak, Ki-Nam Uhm, Kwang-sun Kim, ${ }^{\dagger,, *}$ and Younghoon Lee* \\ Department of Chemistry, KAIST, Daejeon 305-701, Korea. *E-mail: Younghoon.Lee@kaist.ac.kr \\ ${ }^{\dagger}$ Korea Research Institute of Bioscience and Biotechnology (KRIBB), Daejeon 305-806, Korea. *E-mail: sunny06@kribb.re.kr \\ University of Science and Technology (UST), Daejeon 305-350, Korea \\ Received April 7, 2011, Accepted April 11, 2011
}

Key Words : Transcription, Sigma 54, pspA promoter, rnpB terminator

IbsC is a small toxin protein in Escherichia coli, whose expression is repressed by a cis-acting small noncoding RNA, SibC (renamed from RygC or QUAD1c). ${ }^{1,2}$ Overexpression of $\mathrm{IbsC}$ or the absence of SibC transcription induces the expression of both $p s p$ operon ( $p s p A B C D E)$ and $p s p G$ gene encoding phage shock proteins, ${ }^{1}$ whose expression were known to be induced by multiple environment stresses or agents such as filamentous phage infection, osmotic shock, continued incubation in stationary phase, heat shock, and ethanol treatment. ${ }^{4,5}$ Although exogenous overexpression of IbsC leads to cell death, physiological roles of IbsC remain unknown especially because the strain without $i b s C$ gene in the chromosome shows no growth defects. ${ }^{1}$ Considering that only Psp proteins are induced by expression of IbsC, not by other similar small toxin proteins such as $\mathrm{LdrD}$, ShoB, and TisB, ${ }^{1}$ the role of $\mathrm{IbsC}$ might specifically be related to phage shock proteins.

The $p s p$ operon is transcribed from $p s p A$ promoter by RNA polymerase holoenzyme containing the alternative stress-responsive sigma factor $54\left(\sigma^{54}\right) .^{5}$ Transcription from $p s p A$ promoter requires $\mathrm{PspF}$ as an activator protein that binds to the upstream region of the core promoter elements. ${ }^{6,7}$ Furthermore, PspA inhibits the $\sigma^{54}$-dependent $p s p A$ transcription by interacting with $\mathrm{PspF}$ and exerting its negative effects on transcription activation by $\mathrm{PspF}^{8}$ Since the IbsC expression causes the induction of $p s p A$ transcription, it is attempting to see how the signal of IbsC gets transduced into the transcription activation. The mechanism involved in linking $\mathrm{IbsC}$ to $p s p A$ transcription is essential for understanding not only the physiological functions of $\mathrm{IbsC}$, but also the regulation mechanism of transcription from $\sigma^{54}$ dependent promoters in response to environmental stresses. To understand the molecular mechanism of the $p s p A$ induction by $\mathrm{IbsC}$, it is necessary to know which factors are involved in the activation of $p s p A$ transcription under conditions of IbsC expression. Although an in vitro transcription system for $p s p A$ promoter analysis was previously set up, ${ }^{8}$ it is difficult to identify in vitro transcripts because their transcription termination sites are unclear. To overcome the drawback of the previous in vitro transcription system, in this study, we constructed plasmid pPR56 containing a transcription fusion of $p s p A$ promoter and $r n p B$ terminator by replacing the $r n p B$ promoter-containing DNA fragment of pLMd23-wt with the $p s p A$ promoter-containing fragment. ${ }^{9,10}$ The $p s p A$ promoter-containing fragment spanning from -320 through +56 of $p s p A$, which also includes two UAS sequences (UAS I and UAS II) as PspF-binding sites and the IHF binding site, ${ }^{6}$ was subcloned into the BamHI/ EcoRI linearlized pLMd23-wt not having the $r n p B$ promotercontaining DNA fragment, to generate the fusion plasmid pPR56 (Fig. 1). The $r n p B$ terminator region in the fusion construct contained the $r n p B$ sequence from +331 to +1286 , which includes the three $r n p B$ terminators T1, T2, and T3 leading to transcription termination at $+413,+526$, and +638 , respectively. Therefore, this pPR56 construct was designed to generate $p s p A-r n p B$ fusion transcripts of $146 \mathrm{nt}$ terminating at T1 (pspAT1), $259 \mathrm{nt}$ at T2 (pspAT2), and 371 nt at T3 (pspAT3) if transcription starts at the transcription initiation site of $p s p A$. Using supercoiled plasmid pPR56 DNA as a template, in vitro transcription was carried out by adding $\mathrm{E} \sigma^{54}$ and PspF. The pspAT1 transcript of $145 \mathrm{nt}$ was produced as a major band although minor pspAT2 and pspAT3 products were also observed (Fig. 2). The increased abundance of three transcripts was observed with the incremental amount of PspF protein, while RNA I ${ }^{11}$ of 108 nt (a transcript transcribed from its own $\sigma^{70}$ specific promoter of the plasmid DNA) was not produced. In contrast, the same transcription reaction performed with $\mathrm{E} \sigma^{70}$ did not produce the $p s p A T$ transcripts. Instead, this reaction generated RNA I. All our data confirmed that the $p s p A$ transcripts were produced from the $\sigma^{54}$-specific $p s p A$ promoter of pPR56. To

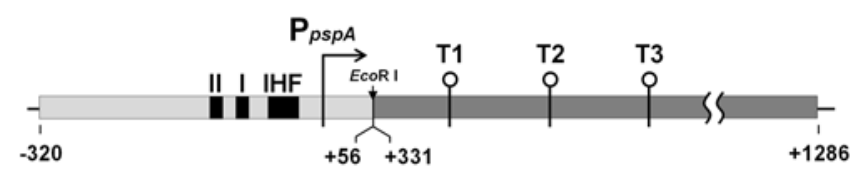

Figure 1. Schematic representation of pPR56 transcription fusion. The $p s p A$ promoter region $(-320$ to +56$)$ was fused to the $r n p B$ terminator region $(+331$ to +1286$)$. Three transcripts are possible from this construct by transcription termination at +413 (T1), +526 (T2), and +638 (T3): pspAT1 of $145 \mathrm{nt}$, pspAT2 of $258 \mathrm{nt}$, and pspAT3 of $371 \mathrm{nt}$. The arrow indicates the transcription start site of $p s p A(+1) . \mathrm{P}_{p s p A}, p s p A$ promoter; I and II, UAS I and II as PspF binding sites; IHF, IHF binding site; E, the 7-bp EcoRI linker as the fusion site. 


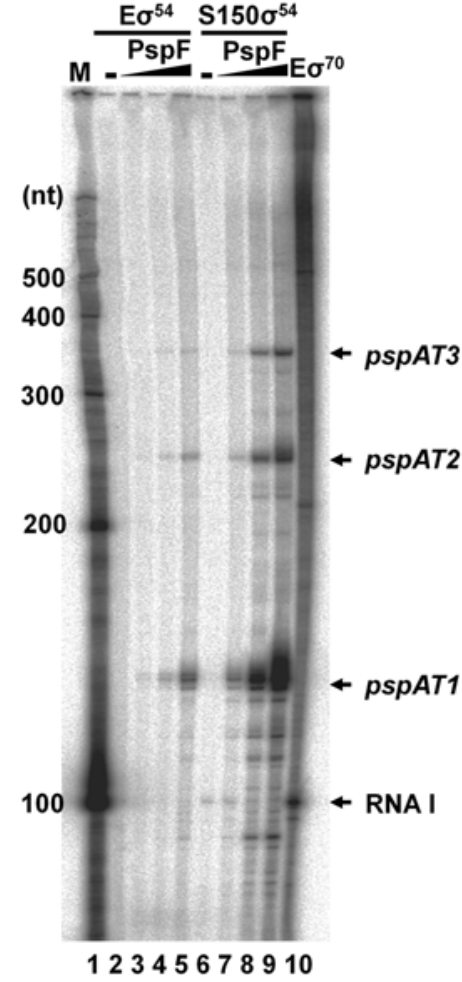

Figure 2. Analysis of in vitro transcription from the $p s p A$ promoter. Plasmid pPR56 DNA was used as a template for in vitro transcription either $\mathrm{E}^{54}$ (lanes 2-5) or $\mathrm{S} 150$ supplemented with $\sigma^{54}$ (lanes 6-9). In vitro transcription reactions were carried out in the presence of PspF protein. The amounts of PspF added in the transcription reactions were 0 (lanes 2 and 6), 4 (lanes 3 and 7), 40 (lanes 4 and 8), and $400 \mathrm{nM}$ (lanes 5 and 9). The resulting RNA products were analyzed in a polyacrylamide sequencing gel. Three transcripts ( $p s p A T 1$ of $145 \mathrm{nt}$, pspAT2 of $258 \mathrm{nt}$, and pspAT3 of 371 nt) are indicated. The same in vitro transcription was carried out with $\mathrm{E \sigma}^{70}$ (lane 10) and the $E \sigma^{70}$-derived product RNA I is indicated. $\mathrm{M}$, size marker (lane 1).

search factors involved in the $p s p A$ induction by $\mathrm{IbsC}$, in vitro transcriptional analysis with extracts from cells grown under the IbsC expression condition is necessary. Therefore, we prepared an $\mathrm{S} 150$ fraction from $E$. coli lysates as an in vivo-mimic transcription machinery for the $p s p A$ induction by IbsC. When $\sigma^{54}$ and PspF were added into the $\mathrm{S} 150$ fraction, $p s p A T$ transcripts were produced and their production was increased with the increasing amount of PspF, suggesting that the $\mathrm{S} 150$ fraction can be used as an in vivo mimicry for analysis of $p s p A$ induction by $\mathrm{IbsC}$.

In conclusion, our results show that the pRS56 fusion was appropriately constructed for efficient analysis of in vitro transcription from the $\sigma^{54}$-specific $p s p A$ promoter. The in vitro transcription system including the utilization of S100 we set up in this study can be used to analyze factors involved in transcription induction of $p s p A$ by $\mathrm{IbsC}$.

\section{Experimental Section}

Construction of a Template DNA for Analysis of Transcription from pspA Promoter. A fusion plasmid contain- ing both $p s p A$ promoter- and $r n p B$ terminator-regions was constructed from plasmid pLMd23-wt, which was a derivative of pGEM3 (Promega) carrying the $r n p B$ transcription unit. $^{10}$ The BamHI-EcoRI fragment of pLMd23-wt was replaced with a $p s p A$ promoter-containing DNA fragment. The promoter-containing DNA fragment was obtained by PCR with a primer pair of BHIpspA-320 (5'-CGC GGA TCC GCA GTT AAG GGA AAT AAA CG-3') and ERIpspAre+56 (5'-CCG GAA TTC AGAA AAA ATA CCC ATA ATG TTG-3'). The PCR products were then digested with BamHI and EcoRI, and cloned into the BamHI-EcoRI site of pLMd23-wt to generate the fusion plasmid pPR56, carrying the promoter regions from -320 to +56 of $p s p A$. For the plasmid construction and propagation, E. coli JM109 was used as the bacterial host strain. ${ }^{12}$

In vitro Transcription by E.coli Polymerase. Plasmid pPR56 DNA was used as a template for in vitro transcription. In vitro transcription reaction was carried out basically as described previously, ${ }^{2}$ with minor modification. Proteins $\sigma^{54}$ and PspF were purified using the corresponding clones from the ASKA library, ${ }^{13}$ as previously described. ${ }^{2} \mathrm{E}^{54}$ was prepared either by combining the core enzyme and $\sigma^{54}$ in a ratio of $1: 4$ or by supplementing $\sigma^{54}$ to $S 150$ fraction (containing RNA polymerase core). For preparing the S150 fraction, S30 fraction from E. coli strain MG1655 prepared as described previously ${ }^{14}$ and the $\mathrm{S} 30$ fraction was further subjected to ultracentrifugation for $2 \mathrm{~h}$ at $4{ }^{\circ} \mathrm{C}$ at $150,000 \times g$. The S150 fraction was used as a crude RNA polymerase. Briefly, $\mathrm{E}^{54}$ (the core enzyme of $90 \mathrm{nM}$ ) or S150 fraction (about $0.3 \mu \mathrm{g} / \mu \mathrm{L}$ protein) supplemented with $\sigma^{54}$ of $360 \mathrm{nM}$ was incubated at $37^{\circ} \mathrm{C}$ for $5 \mathrm{~min}$ in the reaction buffer $(40$ $\mathrm{mM}$ Tris- $\mathrm{HCl}, \mathrm{pH} 8.0,10 \mathrm{mM} \mathrm{MgCl} 2,10 \mathrm{mM}$ DTT, 150 $\mathrm{mM} \mathrm{KCl}, 0.05 \%$ Triton X-100) with $6 \mathrm{nM}$ of template DNA and $40 \mathrm{mM}$ rATP. The reaction was started by adding rNTP mixtures $(500 \mu \mathrm{M}$ of GTP, UTP, and $25 \mu \mathrm{M}$ of CTP including $10 \mu \mathrm{Ci}$ of $\left.\left[\alpha-{ }^{32} \mathrm{P}\right] \mathrm{CTP}\right)$ in the presence of varying amounts of PspF protein (0 to $400 \mathrm{nM}$ ). After $25 \mathrm{~min}$, the reaction was terminated by the addition of the same volume of phenol:chloroform (5:1) mixture and the products were ethanol-precipitated. In vitro transcription reaction with $\mathrm{E}^{70}$ (Epicentre) was also conducted as described above except for using 1 unit of $\mathrm{E \sigma}^{70}$. The products were analyzed on a $5 \%$ polyacrylamide sequencing gel containing $7 \mathrm{M}$ urea and quantitated by BAS1500 (Fuji).

Acknowledgments. We thank the National Institute of Genetics (Shizuoka, Japan) for providing us the ASKA plasmids. This research was supported by a grant from the Korea Research Foundation and the Korean Government (MOEHRD, Basic Research Promotion Fund) (KRF 2008313-C00542) and the National Research Foundation of Korea (NRF) grant by the Korea government (MEST) (2010-0029167).

\section{References}

1. Fozo, E. M.; Kawano, M.; Fontaine, F.; Kaya, Y.; Mendieta, K. S.; 
Jones, K. L.; Ocampo, A.; Rudd, K. E.; Storz, G. Mol. Microbiol. 2008, 70, 1076

2. Han, K.; Kim, K. S.; Bak, G.; Park, H.; Lee, Y. Nucleic Acids Res. 2010, 38, 5851

3. Darwin, A. J. Mol. Microbiol. 2005, 57, 621.

4. Kobayashi, R.; Suzuki, T.; Yoshida, M. Mol. Microbiol. 2007, 66, 100.

5. Weiner, L.; Brissette, J. L.; Model, P. Genes Dev. 1991, 5, 1912.

6. Jovanovic, G.; Model, P. Mol. Microbiol. 1997, 25, 473.

7. Weiner, L.; Brissette, J. L.; Ramani, N.; Model, P. Nucleic Acids Res. 1995, 23, 2030
8. Dworkin, J.; Jovanovic, G.; Model, P. J. Bacteriol. 2000, 182, 311.

9. Ko, J.; Lee, S. J.; Cho, B.; Lee, Y. FEBS Lett. 2006, 580, 539.

10. Kim, S.; Kim, H.; Park, I.; Lee, Y. J. Biol. Chem. 1996, 271, 19330.

11. Jung, Y. H.; Lee, Y. Mol. Biol. Rep. 1996, $22,195$.

12. Yanisch-Perron, C., Vieira, J.; Messing, J. Gene 1985, 33, 103.

13. Kitagawa, M.; Ara, T.; Arifuzzaman, M.; Ioka-Nakamichi, T.; Inamoto, E.; Toyonaga, H.; Mori, H. DNA Res. 2005, 12, 291.

14. Roberts, H. D.; Altman, S.; Smith, J. D. J. Biol. Chem. 1972, 247 , 5243. 